\title{
Sequences in locally convex spaces
}

by

J. K. HAMPSON and A. WILANSKY (Bethlehem, Pa.)

Abstract. An example is given of a non-semibornological $O$-sequential space. It is remarked that the fact that the dual of a Mazur space is strongly complete improves a recent result on spaces with a basis.

We give an example, Example 3, to disprove the assertion made in [7] p. 52, fortunately without proof, that a $C$-sequential locally convex space must be semibornological. In the course of further discussion of sequences, we are also able to give a strong improvement of a result of 0 . T. Jones [1], using a slight generalization of a theorem in [3].

By $E$ we designate a fixed locally convex topological vector space, [3], Chapter 4 ; [6], Chapter $12 ; E^{\prime}$ is its dual, $E^{s}$ is the set of sequentially continuous linear functionals, i.e. linear $f: E \rightarrow$ complex numbers satisfying $f\left(x_{n}\right) \rightarrow 0$ whenever $\left\{x_{n}\right\}$ is a null sequence in $E ; E^{b}$ is the set of bounded linear functionals i.e. such that $f[B]$ is bounded whenever $B$ is. We call $E$ a Mazur space whenever $E^{s}=E^{\prime}$, and semibornological whenever $E^{b}=E^{\prime}$. Clearly $E^{\prime} \subset E^{8} \subset E^{b}$ ([7], p. 52) so that semibornological implies Mazur. A set $U$ is called a sequential neighborhood of 0 if every null sequence belongs to $U$ eventually, i.e. $x_{n} \rightarrow 0$ implies that $x_{n} \in U$ for all sufficiently large $n ; E$ is called $C$-sequential if every convex sequential neighborhood of 0 is a neighborhood of 0 .

By $w^{*}$ we mean $\sigma\left(E^{\prime}, E\right)$, the weak star topology on $E^{\prime}$. A linear functional $f$ on $(E, T)^{\prime}$ is called aw* continuous if $f \mid M$ is $w^{*}$ continuous

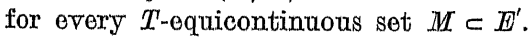

LEMvis 1. $E$ is $C$-sequential if and only if every absolutely convex sequential neighborhood of 0 is a neighborhood of 0 .

To prove sufficiency, let $U$ be a convex sequential neighborhood of 0 . Let $\dot{V}=U \cap(-U)$ and $W=V \cap(i V)$. For $|t| \leqslant \frac{1}{2}, t=r+i s$, We have $\mathrm{tW} \subset r W+i s W \subset \frac{1}{2} W+i \frac{1}{2} W$ (since $W$ is convex and contains 0 ) $c \frac{1}{2} U+\frac{1}{2} U=U$. Let $H$ be the convex hull of $\bigcup\left\{t W:|t| \leqslant \frac{1}{2}\right\}$. The above remarks imply that $H \subset U$. Now $H$ is absolutely convex and, since $H \supset \frac{1}{2} W$, it is a sequential neighborhood of 0 , hence a neighborhood of 0 , and so $U$ is also. 
Now if $T$ is the topology of $E$, Webb [5] denotes by $T^{+}$the largest locally convex topology for $E$ which has the same convergent sequences as $T$. A base for the $T^{+}$neighborhoods of 0 is the set of all absolutely convex sequential neighborhoods of 0 .

Lemma 2. $T^{+}$is $C$-sequential.

This is immediate from Lemma 1.

LEMMA 3. Let $(E, T)$ be a locally convex space such that $\left(E^{\prime}, w^{*}\right)$ is a Mazur space. The w*t is compatible with the duality $\left(\mathbb{E}^{\prime}, \mathbb{E}\right)$ i.e. $\left(\mathbb{E}^{\prime}, w^{*+}\right)^{\prime}$ $=E$.

First $w^{*+} \supset w^{*}$ so that the dual includes $\mathbb{E}$. Next let $f$ be $w^{*+1}$ continuous on $\mathbb{E}^{\prime}$. Then $f$ is $w^{*}$ sequentially continuous, hence $w^{*}$ continuous, so that $f \in E$.

The next result is essentially [3], 21.9 (5).

Lemma 4. Let $E$ be separable and complete. Then $\left(\mathbb{E}^{\prime}, w^{*}\right)$ is a Mazur space.

Let $f$ be $w^{*}$ sequentially continuous and $M$ a $T$-equicontinuous set in $E^{\prime}$. Then $\left(M, w^{*}\right)$ is metrizable, $([3], 21.3(4))$ so $f \mid M$ is continuous i.e. $f$ is aw* continuous. By [2], 16.9, $f$ is $w^{*}$ continuous.

EXAMPLE 1. "Separable" cannot be dropped in Lemma 4 even when $t$ is a Banach space, [4].

EXAMPLLE 2. "Oomplete" cannot be dropped in Lemma 4 even when $E$ is a separable normed space. Let $(E, T)$ be barreled, [7], p. 53, or more generally sequentially barreled in the sense of [5]. Then every aw* continuous linear functional is $w^{*}$ sequentially continuous since every $w^{*}$ convergent sequence is $T$-equicontinuous, but need not be $w^{*}$ continuous, [2], 16.9 .

The next result is seentially [2] 20A. By $\tau$ we mean the Mackey topology $\tau\left(E^{\prime}, E\right)$

Lemma 5. Let $E$ be a Banach space such that $\left(\mathbb{E}^{\prime}, \tau\right)$ is bornological. Then $E$ is reflexive.

For $\tau$ has the same bounded sets as the norm topology on $\mathbb{E}^{\prime}$ hence is larger. Thus these topologies are equal. (See [6], 7.6, Theorem $1 ; 10.5$, Problem 23.)

EXAMPLE 3. A 0 -sequential locally convex space which is not semibornological. Let $E$ be a separable non-reflexive Banach space and $X$ $=\left(\mathbb{E}^{\prime}, w^{*+}\right)$. By Lemma $2, X$ is $O$-sequential. Now suppose that $X$ is semibornological. By Lemmas $3,4,\left(\mathbb{E}^{\prime}, \tau\right)$ is semibornological since all compatible topolgies have the same bounded sets, [6], 12.3, Theorem 1. By [3] $28.1(3)$, or [2], 19.4, $\left(E^{\prime}, \tau\right)$ is bornological, contradicting Lemma 5.

THEOREM 1. Let $E$ be a Mazur space. Then $\mathbb{E}^{\prime}$ is strongly complete.
This follows, with slight modifications, from the proof given in [3], 21.6 (4) which assumes $\mathbb{E}$ metrizable. Note that this also implies the same result if $E$ is bornological, which is given, with a different proof in [3], 28.5 (1). Note also that we get the stronger result that $E^{\prime}$ is complete with the topology of uniform convergence on null sequences in $E$.

Theorem 1 is a significant generalization of Theorem 6 of [1]. This result deals with spaces with a schauder basis $\left\{x^{n}\right\}$ and biorthogonal $\left\{f_{n}\right\}$. Jones proves that if every linear functional $g$ satisfying $g(x)$ $=\sum f_{n}(x) g\left(x^{n}\right)$ for all $x$ is continuous, then $E^{\prime}$ is strongly complete. Since the given condition implies that $E$ is a Mazur space, Theorem 1 applies, as well as the stronger result mentioned in its proof. The next result, with Theorem 1, has, as a special case, the remark of [1] that the strong dual of a barreled space with Schauder basis is complete. It is well known that this is false without the basis assumption; hence also, a barreled space need not be a Mazur space.

THEOREM 2. Let $\mathbb{E}$ be a barreled space with a Schauder basis. Then $E$ is a Mazur space.

If $g$ is sequentially continuous we have $g(\dot{x})=\sum f_{n}(x) g\left(x_{n}\right)$ and so $g$ is continuous by the Banach-Steinhaus closure theorem, [6], Section 12.3, Theorem 5 .

In [7], pp. 50-61, are given some connections among the various properties listed above. In addition we note that a Mazur space need not be semibornological as is shown by Lemmas 4,5 and the fact that "semibornological" is a duality invariant.

\section{References}

[1] O. T. Jones, Oontinuity of seminorms and linear mappings on a space with Schauder basis, Studia Math. 34 (1970), pp. 121-126.

[2] J. L. Kelley and I. Namioka, Linear topological spaces, 1963.

[3] G. Köthe, Topological vector spaces I. 1969.

[4] B. Kripke, One more reason why sequences are not enough, Amer. Math. Monthly 74 (1967), pp. 563-565.

[5] J. H. Webb, Sequential convergenoe in locally oonvex spaces, Proc. Camb. Phil. Soc. 64 (1968), pp. 34:1-363.

[6] A. Wilansky, Functional analysis, 1964

[7] - Topios in functional analysis, 1967.

LEHIGE UNIVERSTTY 